\title{
Statistik für alle - Die 101 wichtigsten Begriffe anschaulich erklärt.
}

Walter Krämer

Springer Spektrum, Berlin, Germany, 2015.

ISBN 978-3-662-45030-7. 89-90 pp. EUR 15.41.

http://www . springer. com/de/book/9783662450307

Zur Vorbeugung von Missverständnissen gleich mal vorne weg: 1. Ja, Walter Krämer hat mich mit seinem in den 90er Jahren erstmals erschienen Buch „So lügt man mit Statistik“ nachhaltig in der Lehre inspiriert; 2. Sein Zugang, die statistische Methodik möglichst anschaulich erklären zu wollen, viel bei mir auf fruchtbaren Boden; und schließlich 3. Ich traue mir dennoch zu, sein neues Buch „Statistik für alle - Die 101 wichtigsten Begriffe anschaulich erklärt“, objektiv zu rezensieren.

Die 101 wichtigsten Begriffe der Statistik also! - Die Auswahl dieser Schlagworte erfolgte natürlich nach der subjektiven Einschätzung des Autors und darüber ließe sich schon mal treffend streiten: Warum sind etwa die Begriffe „Benford-Gesetz“, „Biometrischer Fingerabdruck“, „Chaos" oder „Itemanalyse“ Bestandteil dieser Auswahl, nicht aber "Inflationsrate“, „p-Wert", „Repräsentativität" oder „Stichprobenumfang“? - Weil es die Auswahl des Autors ist, möchte man antworten, und als solche ist sie natürlich die richtige.

Die Begriffe sind wie in einem Wörterbuch alphabetisch geordnet von „Achsenmanipulation“ bis „Zufallszahlen“. Es querzulesen bzw. gezielt nachzuschlagen erschiene mir deshalb eigentlich als geeigneter Zugang zur Lektüre. Dabei muss festgehalten werden, dass zu diesem Zweck so manche außerhalb des Faches eher nicht so bekannten Begriffe wie beispielsweise „,arithmetisches", „geometrisches" oder „harmonisches" Mittel vielleicht doch besser in einem Oberbegriff wie „Mittelwert“ zusammengefasst worden wären, damit man schließlich auch auf diese Fachbegriffe, quasi en passant, stoßen kann, ohne bewusst danach suchen zu müssen.

Mein Zugang als Rezensent war es dennoch, es von vorne nach hinten zu lesen. Krämer schreibt gewohnt sachlich korrekt, dabei aber um allgemeine Verständlichkeit bemüht. Ein Beispiel dafür ist der Eintrag zur „Binomialverteilung“. Diese motiviert Krämer an Hand der Frage: „An wieviel Börsentagen in der Woche schließt der DAX im Plus? ... Die Binomialverteilung sagt: wir können uns dieses Auszählen sparen." Und er merkt dabei auch noch an, dass die von ihm mit 1/2 angesetzte Ereigniswahrscheinlichkeit ,in Wahrheit ein kleines bisschen größer" als $1 / 2$ ist. Ab und an wird in den Einträgen pointiert formuliert. Ein Beispiel dafür findet sich bei den Bevölkerungspyramiden unter dem Schlagwort „Histogramm“: „Man sieht sehr schön die jetzt 45-55-jährigen Babyboomer der Geburtsjahrgänge 1958-1967, die gerade in vollen Zügen ihre historische Ausnahmesituation geniessen: wenige Kinder, die man unterhalten muss, und sehr viele Geschwister, mit denen man sich den Unterhalt der Eltern teilt." Zuweilen sind die Einträge auch polemisch gewürzt. Unter dem Terminus „Arbeitslosenquote“ folgert Krämer beispielsweise: „Demnach sind Teilnehmer von Schul- und Umschulungsmaßnahmen, da nicht unmittelbar dem Arbeitsmarkt zur Verfügung stehend, offiziell nicht arbeitslos. ... Unter anderem auch deshalb sind die jeweils regierenden und an niedrigen Arbeitslosenzahlen interessierenden Kreise so große Freunde von Langzeitstudenten und Umschulungsprogrammen aller Art." 
Das Buch wendet sich meines Erachtens vordergründig nicht an professionelle Statistiker, wenngleich auch solche durchaus den ein oder anderen interessanten Abschnitt darin finden werden. Also gab ich es nach meiner Lektüre kurzerhand an meine Frau weiter - eine interessierte, beruflich sich immer wieder mit Statistiken auseinandersetzen müssende Nichtstatistikerin. Und siehe da: Ich sah sie von Zeit zu Zeit schmunzeln, zustimmend nicken und das ein oder andere Mal diskutierte sie das eben Nachgeschlagene mit mir.

Meine Schlussfolgerung aus diesen Erfahrungen lautet: Das Lesen dieses statistischen WörterBüchleins wird jenen am meisten Freude bereiten und Nutzen bringen, die sich, selbst Nichtstatistiker, aus unterschiedlichen Gründen - sei es beruflich oder im Studium - mit dem Fach auseinandersetzen müssen. Für diese Leserschaft ist es absolut lesenswert, lehrreich und auch launig geschrieben - ein Krämer eben! Ob die Statistik und ihre Anwendung in unserem Leben in 101 Stichwörtern tatsächlich nur Walter Krämer kurz, prägnant und verständlich erklären kann, wie es der Verlag dem Taschenbuch auf den Rücken gedruckt hat, möchte ich dahingestellt lassen.

\section{Reviewer:}

Andreas Quatember

IFAS - Institut für Angewandte Statistik

Johannes Kepler Universität Linz

Österreich E-mail: andreas.quatember@jku.at

\section{Austrian Journal of Statistics}

published by the Austrian Society of Statistics

Volume 46

February 2017 http://www.ajs.or.at/

http://www.osg.or.at/

Published: 2016-11-09 